\title{
A NEW RECORD OF Impatiens kamtilongensis Toppin (Balsaminaceae) FOR VIETNAM FLORA
}

\author{
Vu Tien Chinh ${ }^{1 *}$, Nguyen Thi Thanh Huong ${ }^{1}$, Bui Hong Quang ${ }^{1}$, Piyakaset Suksathan ${ }^{2}$ \\ ${ }^{1}$ Institute of Ecology and Biological Resources, VAST, *tienchinhvu@gmail.com \\ ${ }^{2}$ Queen Sirikit Botanic Garden, Chiang Mai Thailand
}

\begin{abstract}
Impatiens kamtilongensis Toppin (Balsaminaceae), collected from Xuan Lien district, Thanh Hoa province is reported here as a new record for the flora of Vietnam. I. kamtilongensis can be distinguished from its closest ally I. finetii by its hairy white stem, yellow flowers having 2 red blotches, bigger sepals and glabrous capsule. The specimens are deposited in the Herbarium of the Institute of Ecology and Biological Resources (HN) Ha Noi, Vietnam. Morphological redescription and illustrations are provided along with notes on distribution, ecology, phenology and conservation of the species.
\end{abstract}

Keywords: Balsaminaceae, Impatiens, I. kamtilongensis new record, Vietnam.

\section{INTRODUCTION}

The genus Impatiens, comprising approximately 900 species is distributed in tropical to subtropical Asia and Africa while very few species occur in temperate Asia, Europe and North America. In Vietnam, this genus comprises 34 species [11,33].

During a recent fieldwork in Xuan Lien Nature Reserve, Northern Vietnam, the first and third author collected and found I. kamtilongensis.

Morphologically, I. kamtilongensis is close to $I$. finetii but it can be distinguished from the later by its hairy white stem and yellow flowers with 2 red blotches.

\section{MATERIALS AND METHODS}

All specimens deposited in various herbaria of Vietnam namely HN, VNM, HNU, NHNPI along with two Chinese herbaria, viz. KUN \& SCBG were studied. Specimens in the virtual herbaria, such as PE, K \& P were also studied. The collected specimens of I. kamtilongensis were thoroughly processed using standard preservation methods and deposited in the herbarium of Institute of Ecology \& Biological Resources (HN).

\section{RESULTS AND DISCUSSION}

Impatiens kamtilongensis Toppin Bull. Misc. Inform. Kew 1920 (10): 356 (1920); Chen,
Akiyama \& Ohba in Fl. China, 2007. 12: 50. Syntype: Myanmar, Kumtat 1600' Sinar 1950' Kachin Hills, Dec. 1911, Toppin 4275 (K photo!).

Procumbent herb, 25-40 cm high, pubescent. Leaves alternate, somewhat congested towards the upper part of stems; lamina elliptic to ovate 4-11 $\times 2.1-4.5 \mathrm{~cm}$, apex acuminate, base cuneate, crenulate to serrate along margins, sparsely pubescent on both surface, chartaceous, basal glands absent or minute projections; lateral veins $7-8$ pairs; petiole up to $3.8 \mathrm{~cm}$ long. Flowers yellow with 2 red round blotches on upper lateral petals, solitary without peduncle, or in a pair with peduncle of ca. $2 \mathrm{~cm}$ long, pedicels 1-2 cm long. Sepals 2, ovate, green, hairy white, suborbicular, 1.1-1.3 cm across, apiculate, pubescent on outer surface; lower sepals yellow, saccate, $1.3-2 \mathrm{~cm}$ long, inner surface glabrous, 2 with or without pale red reticulate-veins, outer surface pubescent, spur entirely yellow, 2-2.5 cm long, downward curved; dorsal petals yellow, ovate oblong, 1.6 $\times 1.4 \mathrm{~cm}$, apex sometimes emarginated, costa broadly winged on the lower half ca. $5 \mathrm{~mm}$ wide; lateral united petals free, lower petals yellow, narrowly obovate, ca. $12 \times 7 \mathrm{~mm}$, upper petals yellow with one red blotch at the middle of the lobe, subtriangular, ca. $13 \mathrm{~mm}$ long. Ovary glabrous. Capsule ellipsoid, ca. $1.3 \mathrm{~cm}$ long, glabrous. Seeds up to 12, rounded, 3-4 $\mathrm{mm}$ long. Fl. May to June; Fr. July. 
Distribution: Vietnam: Thanh Hoa (Xuan Lien, Thuong Xuan); Myanmar (Kachin); South China (Yunnan).

Ecology and habitat: The species was found growing in shade nearby a stream in evergreen forest of limestone hills at about $1,248 \mathrm{~m}$ alt.

Specimens examined: Northern Vietnam, Thanh Hoa province, Thuong Xuan district, Xuan Lien Nature Reserve, 27 June 2013, Chinh 26; 05 July 2014, Quang 91 [HN].

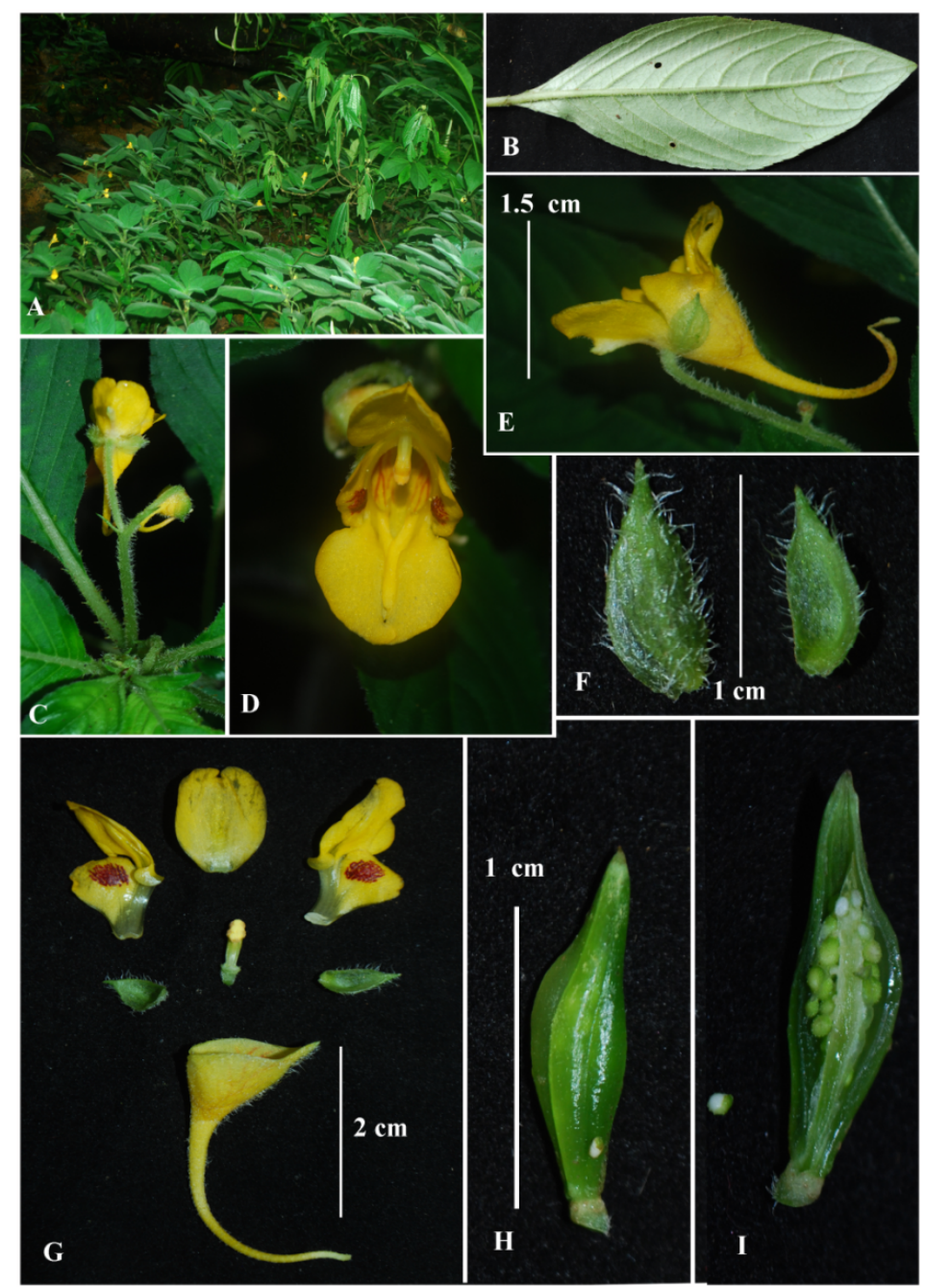

Figure 1. Impatiens kamtilongensis Toppin

A. habit; B. leaf;

C. inflorescence;

D. flower-front view;

E. flower-lateral view;

F. calyx;

G. flower-dissected;

H. fruit;

I. open fruit showing seeds.

Photographed by Bui Hong Quang.

Table 1. Morphological comparison of I. kamtilongensis with I. finetii

\begin{tabular}{cll}
\hline Morphological characters & \multicolumn{1}{c}{ I. kamtilongensis } & \multicolumn{1}{c}{ I. finetii } \\
\hline Stem & hairy white & hairy red \\
Leaves & $7-8$ pairs & $9-10$ pairs \\
Petiole & $2-3.8 \mathrm{~cm}$ long & ca. $5 \mathrm{~cm}$ long \\
Sepals & $1.1-1.3 \mathrm{~cm}$ long; saccate, $1.3-2 \mathrm{~cm}$ & ca. $0.7 \mathrm{~cm}$ long; saccate, ca. $3 \mathrm{~cm}$ \\
Petals & ca. $1.6 \mathrm{~cm}$ long. & ca. $1.2 \mathrm{~cm}$ long. \\
Capsule & ca $1.3 \mathrm{~cm}$ long, glabrous & ca $1 \mathrm{~cm}$ long, hairy \\
\hline
\end{tabular}




\section{CONCLUSION}

I. kamtilongensis is reported here as a new record for the flora of Vietnam.

I. kamtilongensis species is morphologically the closest ally of $I$. finetii (table 1). This species has some striking difference in morphological characteristics, such as, lateral 78 pairs, petiole $2-3.8 \mathrm{~cm}, 2$ red round blotches on their upper lateral petals (fig. 1). Present report will help prioritizing conservation of this species in Vietnam, China and Myanmar.

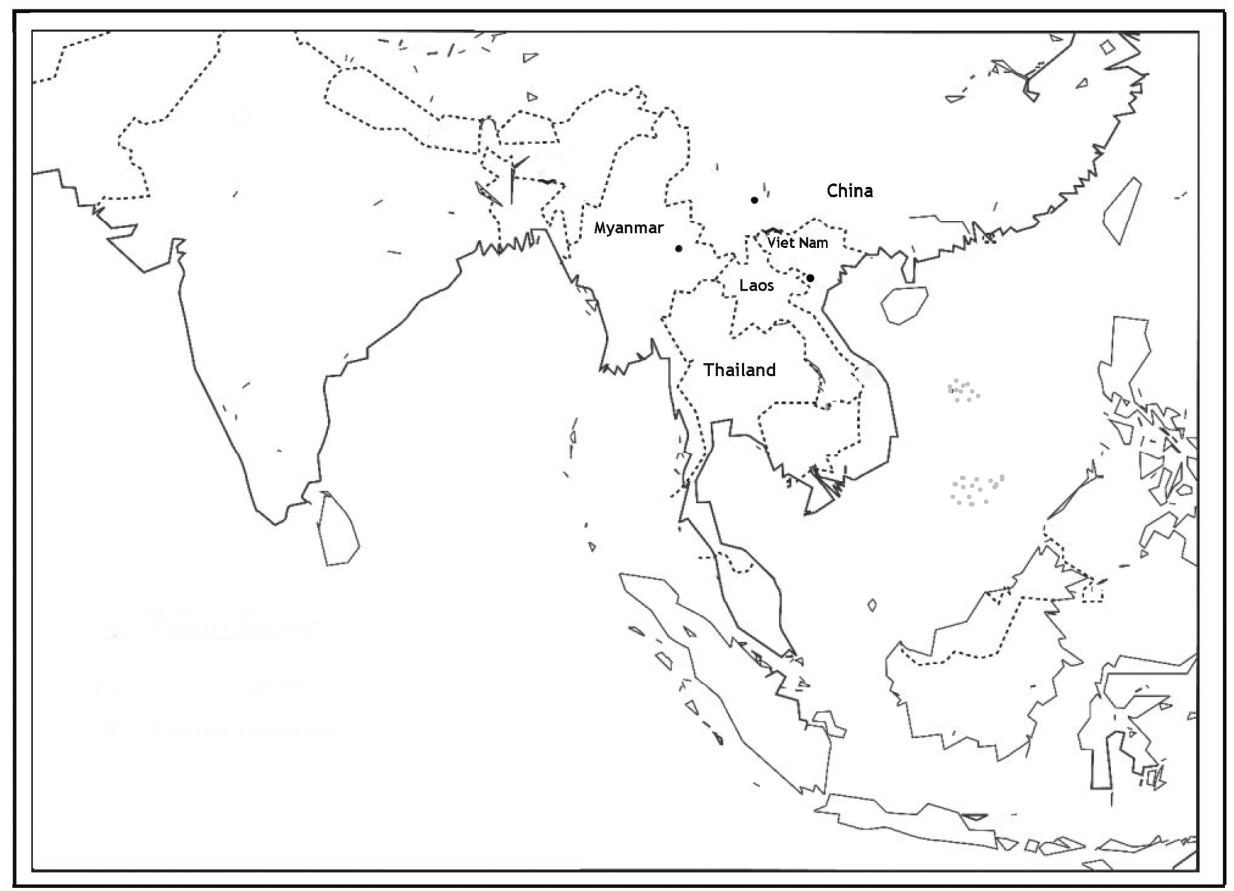

Figure 2. Distribution map of Impatiens kamtilongensis Toppin showing its localities in the world

Acknowledgments: We are thankful to the authorities of Institute of Ecology and Biological Resources (IEBR), Vietnam Academy of Science and Technology Ha Noi (VAST). This research was funded by Vietnam, Institute of Ecology and Biological Resources vide project no. IEBR.DT01/15-16.

\section{REFERENCES}

1. Nguyễn Tiến Bân, 2003. Danh lục các loài thực vật Việt Nam (Checklist of Plant Species of Vietnam), 2: 1047-1051. Nxb. Nông nghiệp, Hà Nội.

2. Gagnepain F., 1946. Balsaminaceae, Supplément à la Flore Générale de 1'IindoChine, 3: 589-592. Paris.

3. Phạm Hoàng Hộ, 1999. Cây cỏ Việt Nam
(An Illustrated Flora of Vietnam), 2: 299236. Nxb. Trẻ, tp. Hồ Chí Minh.

4. Lecomte H., 1911. Balsaminaceae, Flore Generale de Indo-Chine, 1(3): 611-629. Paris.

5. Linnaeus C., 1753. Impatiens, Species Plantarum, 2: 937-238.

6. Newman M., Ketphanh S., Bouakhay K., Thomas P., Khamphone S., Lamxay V., And Kate A., 2007. Checklist of the vascular Plants of Lao PDR. Edinburgh: Royal Botanic Garden. pp. 73.

7. IUCN, 2012. IUCN Red List Categories and Criteria: Version 3.1. Prepared by the IUCN Species Survival Commission. IUCN, Gland, Switzerland, and Cambridge, United Kingdom. 
8. Yilin C., Shinobu A., \& Hideaki O., 2007. Balsaminaceae, Flora of China, in Wu C. Y., \& Raven P. H., (Eds.), Vol. 12. pp. 12-43. Beijing Science Press \& St. Louis: Missouri Botanical Garden Press.
9. Yu S. X., 2012. Balsaminaceae of China, pp. 72-206. Peking University Press.

10. http://www.theplantlist.org/tpl1.1/record/tro -50151557 (update 5/8/2015).

\title{
BỔ SUNG LOÀI Impatiens Kamtilongensis Toppin (Balsaminaceae) CHO HỆ THỰC VẬT VIẸTT NAM
}

\author{
Vũ Tiến Chính ${ }^{1}$, Nguyễn Thị Thanh Hương ${ }^{1}$, Bùi Hồng Quang ${ }^{1}$, Piyakaset Suksathan ${ }^{2}$ \\ ${ }^{1}$ Viện Sinh thái và Tài nguyên sinh vật, Viện Hàn lâm KH \& CN Việt Nam \\ ${ }^{2}$ Vườn Thực vật Nữ hoàng Sirikit, Chiềng Mai, Thái Lan
}

\section{TÓM TÁ́T}

Phân tích các mẫu thu được từ khu Bảo tồn Xuân Liên, tỉnh Thanh Hóa năm 2014, chúng tôi đã phát hiện loài Impatiens kamtilongensis Toppin bổ sung cho hệ thực vật Việt Nam, mẫu nghiên cứu được Vũ Tiển Chính và Bùi Hồng Quang có số hiệu Chinh 26, Quang 91, được lưu giữ tại phòng tiêu bản Viện Sinh thái và Tài nguyên sinh vật $(\mathrm{HN})$. Bài báo này còn đưa ra hình ảnh minh họa, phân bố và so sánh loài mới được ghi nhận với loài I. finetii.

Loài I. kamtilongensis có các đặc điểm khác biệt với những loài khác trong chi Impatiens ở Việt Nam ở chỗ có hoa màu vàng có hai vòng tròn đỏ. Đài 2 , có lông màu trắng. Lá có 7-8 cặp gân phụ. Mùa ra hoa tháng 5 đến tháng 6 , ra quả tháng 7 đến tháng 8 hàng năm.

Từ khóa: Balsaminaceae, Impatiens, I. kamtilongensis, ghi nhận mới, Việt Nam.

Ngày nhận bài: 15-5-2015 